\title{
A regional approach for CAR T-cell therapy for mesothelioma: from mouse models to clinical trial
}

\author{
"Regional administration of CAR T cells for locoregionally \\ aggressive solid tumors ... provides an opportunity to investigate \\ regional immune interventions that can be effective alone or in \\ combination with systemic immune interventions..."
}

First draft submitted: 11 January 2016; Accepted for publication: 27 January 2016; Published online: 3 May 2016

Keywords: adoptive T-cell therapy • chimeric antigen receptor $\bullet$ mesothelin

\begin{abstract}
Adoptive T-cell therapy using chimeric antigen receptors (CAR) is an emerging form of immunotherapy that harnesses a patient's own T cells to combat malignancies [1]. CAR T-cell therapy uses genetic engineering to express a receptor on $\mathrm{T}$ cells that binds to a tumor-associated antigen and activates T-cell cytotoxicity. T cells transduced with antigenspecific CARs are reinfused to the patient in an effort to target tumors. CAR T-cell therapy has already demonstrated promising results in hematologic malignancies [2]. However, translating this approach to solid tumors faces the additional barriers of T-cell homing and infiltration into the tumor. Published literature that has correlated improved prognosis with antitumor immune responses in solid tumors, including in malignant pleural mesothelioma (MPM) [3-5], underscores the need to investigate this promising therapy in clinically relevant models.
\end{abstract}

\section{Malignant pleural mesothelioma: a regionally aggressive disease}

Malignant pleural mesothelioma is a rare, highly aggressive primary pleural tumor with a median survival of 9-20 months, even after multimodality treatment [6]. In contrast to other solid malignancies whose fatalities are secondary to metastasis, MPM aggressiveness is exerted locoregionally with the majority of morbidity resulting from regional invasion. The localized nature of the disease with rare extrathoracic metastases has motivated researchers to attempt intrapleural delivery of therapeutic agents, including chemotherapy [7], photodynamic therapy [8] and radiation with brachytherapy [9], with limited success. Other localized therapies have sought to enhance antitumor immune responses with intrapleural delivery of oncolytic viruses [10,11], IFN- $\beta$ gene transfer [12], or IL-2 [13], with promising, albeit limited, results. For example, with regards to oncolytic viruses, although immune responses were elicited, viruses were rendered incompetent due to neutralizing antibodies. Nevertheless, regional administration of oncolytic viruses to generate immune response, combined with checkpoint blockade, is an emerging area of investigation for the treatment of MPM.

\section{Clinically-relevant mouse model of MPM}

To investigate regional immune interventions, our laboratory has developed and characterized an orthotopic mouse model of MPM [14] that recapitulates human disease with the hallmarks of chest wall and diaphragmatic invasion, and lung and mediastinal encasement. These tumors demonstrate extensive lymphangiogenesis and mediastinal lymph node metastasis in line with the
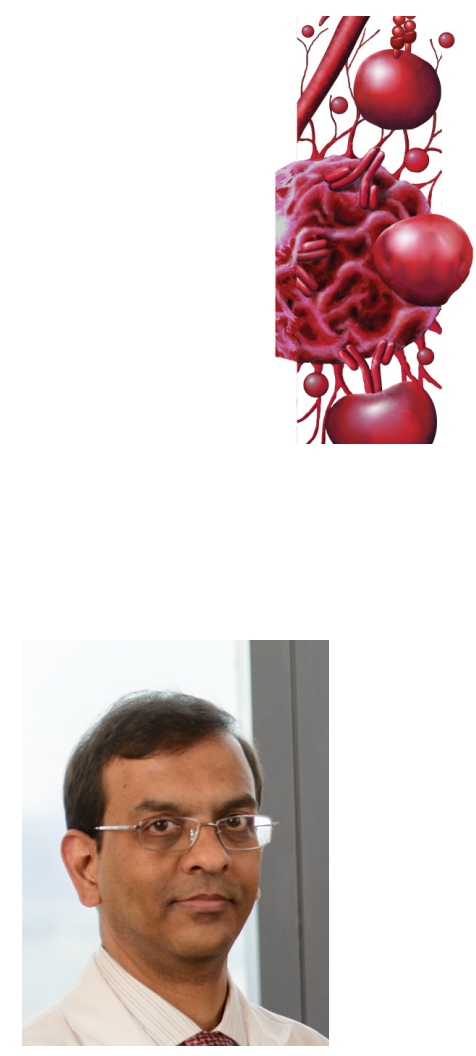

Marissa Mayor ${ }^{1}$, Masha Zeltsman', Erin McGee ${ }^{1}$ \& Prasad S Adusumilli*, 1,2 'Thoracic Service, Department of Surgery, Memorial Sloan Kettering Cancer Center, New York, NY, USA ${ }^{2}$ Center for Cell Engineering, Memorial Sloan Kettering Cancer Center, New York, NY, USA *Author for correspondence: adusumip@mskcc.org 
natural progression of MPM. Furthermore, to monitor tumor burden progression and regression in response to therapy, we optimized noninvasive dual tumor and T-cell imaging protocols $[15,16]$.

\section{Potential for effective immune responses in MPM}

Immunotherapeutic approaches have built upon the findings that a shift of balance from the protumor, immunosuppressive microenvironment that exists within MPM [17] toward an antitumor, effector immune profile may improve prognosis [5]. Our initial investigation of a cohort of 175 MPM patients demonstrated that chronic inflammation in tumor stroma was an independent predictor of overall survival among patients with epithelioid MPM [3]. In a subsequent comprehensive investigation of the tumor immune microenvironment of 230 epithelioid MPM patients, an increased number of CD163+ tumorassociated macrophages with low CD8+ lymphocyte infiltration correlated with worse prognosis and low CD163 + tumor-associated macrophages with high CD20 + lymphocytes correlated with improved prognosis on multivariate analysis [4]. Eliciting immune responses in an effort to tilt the balance toward antitumor activity within the tumor immune microenvironment is a promising area for future investigations.

\section{Ideal antigen target in MPM}

Due to its overexpression in epithelioid MPM, which is the most common type of MPM, and very low levels of expression in normal mesothelia, mesothelin (MSLN) has been investigated as a target antigen. Mesothelin is a glycoprotein that is predominantly expressed on the cell surface of MPM tumor cells and has been shown to promote invasion, both in vitro and in vivo, in an orthotopic MPM mouse model and in MPM patients [18]. Immunotherapeutic agents targeting MSLN, such as SS1P and amatuximab, are currently being evaluated in clinical trials; SS1P has demonstrated safety in Phase I clinical trials [19]. A Phase II clinical trial found that amatuximab, in combination with pemetrexed and cisplatin, resulted in a $40 \%$ partial response with a $51 \%$ stable disease rate in patients with unresectable MPM [20]. Other candidate target antigens currently being investigated for CAR T-cell therapy for MPM includes chondroitin sulfate proteoglycan-4 [21] and fibroblast activation protein [22]; both of these target antigens are currently in the preclinical phase and have shown efficacy in in vitro and in vivo models. In addition to MPM, MSLN is being investigated as a target antigen in other solid tumors [23]. Mesothelin-targeted therapies have demonstrated safety in Phase I clinical trials [19], with no reported on-target, off-tumor toxicities related to MSLN expression on normal tissue.

\section{Regional delivery of CAR T cells overcomes barriers to tumor homing \& infiltration}

In our MPM mouse model, systemically administered CAR T cells are sequestered in the lung with delayed entry into the pleural tumor. Impressively, MSLN-targeted, CD28 co-stimulated (M28z) CAR $T$ cells administered intrapleurally (regional administration) eradicated established pleural tumors, even at a 30-fold lower dose than intravenously administered CAR T cells [16]. Additionally, regionally delivered CAR T cells were able to traffic effectively from the pleural cavity to both flank and peritoneal tumor sites, and they exhibited systemic, long-term, antitumor immunity. T-cell imaging showed that regionally delivered CAR T cells accumulate at higher numbers and at an earlier time point. Further mechanistic investigations demonstrated that early exposure to the antigen via CAR T-cell delivery directly to the tumor location resulted in a robust differentiation into effector phenotypes, as measured by the CD62L(-) subset, compared with intravenously administered $\mathrm{T}$ cells.

Interestingly, in our MPM model, early infiltration and activation of the CD4 + CAR T-cell subset was central to the increase in efficacy seen with regional administration of M28z CAR T cells. In experiments conducted using regionally administered $\mathrm{CD} 4+$ and CD8 + T-cell subsets, independently or together, the CD4+ CAR T-cell subset was independently efficacious; it exhibited comparative, though not equivalent, antitumor efficacy to unsorted, bulk CAR T cells. Additionally, CD4+ CAR T cells exhibited persistence and systemic protection that prevented tumor growth following intraperitoneal challenge at 196 days following a single dose of intrapleural CD4 + CAR T cells. In fact, this early CD4+ subset activation enriched proliferation and in vivo accumulation of CD8+ CAR T cells, thus highlighting the dual functionality of the CD4+ T-cell subset in both helper and cytotoxic activities. Finally, CD28 co-stimulation encoded in our CAR construct proved crucial to antitumor efficacy. Compared with MSLNtargeted CAR T cells without CD28 costimulation, M28z CAR T cells exhibited superior in vivo antitumor efficacy with systemic protection against extrapleural tumor challenge.

Other strategies to enhance CAR T-cell infiltration in MPM included forced expression of chemokine receptors [24] and alternate CAR constructs that used natural multichain immunoreceptor designs [25]. Forced expression of chemokine receptors, such as CCR2, allows CAR T cells to exploit the chemokine 
gradient produced by MPM and to home more effectively to the tumor [24]. Alternate CAR constructs using killer immunoglobulin-like receptors and DAP12 seek to diversify the cytotoxic signaling upon antigen activation [25]. These strategies have demonstrated efficacy in preclinical studies against MPM but have yet to be translated to clinical trials.

\section{Mesothelin-targeted CAR T-cell therapy clinical trials for MPM}

Intravenous delivery of MSLN-targeted CARs was evaluated in a Phase I clinical trial that included three patients with MPM. All three patients in this trial developed an antibody response to the murine component of this CAR, with one patient developing an anaphylactic reaction. No on-target, off-tumor toxicities were reported [26].

Based on our preclinical data, we are conducting a Phase I clinical trial (NCT02414269) to evaluate the safety of regionally administered MSLN-targeted CAR $\mathrm{T}$ cells in patients with either primary pleural malignancy (mesothelioma) or secondary pleural malignancies (lung and breast cancers) with MSLN expression. Our construct is comprised of only human sequences and encodes a suicide gene (i-Caspase-9) that induces apoptosis upon administration of the substrate. In our clinical trial, MSLN-targeted CAR T cells are being administered regionally via an indwelling intrapleural catheter.

\section{References}

1 Sadelain M, Brentjens R, Riviere I. The promise and potential pitfalls of chimeric antigen receptors. Curr. Opin. Immunol. 21(2), 215-223 (2009).

2 Davila ML, Riviere I, Wang X et al. Efficacy and toxicity management of 19-28z CAR T cell therapy in B cell acute lymphoblastic leukemia. Sci. Transl. Med. 6(224), 224 ra225 (2014).

3 Suzuki K, Kadota K, Sima CS et al. Chronic inflammation in tumor stroma is an independent predictor of prolonged survival in epithelioid malignant pleural mesothelioma patients. Cancer Immunol. Immunother. 60 (12), 1721-1728 (2011).

4 Ujiie H, Kadota K, Nitadori JI et al. The tumoral and stromal immune microenvironment in malignant pleural mesothelioma: a comprehensive analysis reveals prognostic immune markers. Oncoimmunology 4(6), e1009285 (2015).

5 Yamada N, Oizumi S, Kikuchi E et al. CD8+ tumorinfiltrating lymphocytes predict favorable prognosis in malignant pleural mesothelioma after resection. Cancer Immunol. Immunother. 59(10), 1543-1549 (2010).

6 Flores RM, Zakowski M, Venkatraman E et al. Prognostic factors in the treatment of malignant pleural mesothelioma at a large tertiary referral center. J. Thorac. Oncol. 2(10),

\section{Conclusion}

Regional administration of CAR T cells for locoregionally aggressive solid tumors, such as MPM, provides an opportunity to investigate regional immune interventions that can be effective alone or in combination with systemic immune interventions (e.g., checkpoint blockade). Knowledge gained from these studies can also be extended to other solid tumors with predominant, locoregional tumor burden.

\section{Acknowledgement}

The authors would like to thank Alex Torres of the MSK Thoracic Surgery Service for his editorial assistance.

\section{Financial \& competing interests disclosure}

This work was supported by grants from the WH Goodwin and A Goodwin, the Commonwealth Foundation for Cancer Research, and the Experimental Therapeutics Center, and the Stand Up To Cancer - Cancer Research Institute Cancer Immunology Translational Cancer Research Grant (SU2C-AACRDT1012). Stand Up To Cancer is a program of the Entertainment Industry Foundation administered by the American Association for Cancer Research. The authors have no other relevant affiliations or financial involvement with any organization or entity with a financial interest in or financial conflict with the subject matter or materials discussed in the manuscript apart from those disclosed.

No writing assistance was utilized in the production of this manuscript.

957-965 (2007).

7 Rusch V, Saltz L, Venkatraman E et al. A Phase II trial of pleurectomy/decortication followed by intrapleural and systemic chemotherapy for malignant pleural mesothelioma. J. Clin. Oncol. 12(6), 1156-1163 (1994).

$8 \quad$ Friedberg JS. Radical pleurectomy and photodynamic therapy for malignant pleural mesothelioma. Ann. Cardiothorac. Surg. 1(4), 472-480 (2012).

9 Hilaris BS, Nori D, Kwong E, Kutcher GJ, Martini N. Pleurectomy and intraoperative brachytherapy and postoperative radiation in the treatment of malignant pleural mesothelioma. Int. J. Radiat. Oncol. Biol. Phys. 10(3), 325-331 (1984).

10 Ranki T, Joensuu T, Jager E et al. Local treatment of a pleural mesothelioma tumor with ONCOS-102 induces a systemic antitumor CD8 T-cell response, prominent infiltration of CD8 lymphocytes and Th1 type polarization. Oncoimmunology 3(10), e958937 (2014).

11 Sterman DH, Treat J, Litzky LA et al. Adenovirus-mediated herpes simplex virus thymidine kinase/ganciclovir gene therapy in patients with localized malignancy: results of a Phase I clinical trial in malignant mesothelioma. Hum. Gene Ther. 9(7), 1083-1092 (1998).

12 Sterman DH, Recio A, Haas AR et al. A Phase I trial of repeated intrapleural adenoviral-mediated interferon-beta 
gene transfer for mesothelioma and metastatic pleural effusions. Mol. Ther. 18(4), 852-860 (2010).

13 Astoul P, Picat-Joossen D, Viallat JR, Boutin C. Intrapleural administration of interleukin-2 for the treatment of patients with malignant pleural mesothelioma: a Phase II study. Cancer 83(10), 2099-2104 (1998).

14 Servais EL, Colovos C, Kachala SS, Adusumilli PS. Preclinical mouse models of primary and metastatic pleural cancers of the lung and breast and the use of bioluminescent imaging to monitor pleural tumor burden. Curr. Protoc. Pharmacol. 14(Unit14), 21 (2011).

15 Servais EL, Suzuki K, Colovos C et al. An in vivo platform for tumor biomarker assessment. PLoS ONE 6(10), e26722 (2011).

16 Adusumilli PS, Cherkassky L, Villena-Vargas J et al. Regional delivery of mesothelin-targeted CAR T cell therapy generates potent and long-lasting CD4-dependent tumor immunity. Sci. Transl. Med. 6(261), 261ra151 (2014).

17 Hegmans JP, Hemmes A, Hammad H, Boon L, Hoogsteden $\mathrm{HC}$, Lambrecht BN. Mesothelioma environment comprises cytokines and T-regulatory cells that suppress immune responses. Eur. Respir. J. 27(6), 1086-1095 (2006).

18 Servais EL, Colovos C, Rodriguez L et al. Mesothelin overexpression promotes mesothelioma cell invasion and MMP-9 secretion in an orthotopic mouse model and in epithelioid pleural mesothelioma patients. Clin. Cancer. Res. 18(9), 2478-2489 (2012).

19 Hassan R, Sharon E, Thomas A et al. Phase 1 study of the antimesothelin immunotoxin SS1P in combination with pemetrexed and cisplatin for front-line therapy of pleural mesothelioma and correlation of tumor response with serum mesothelin, megakaryocyte potentiating factor, and cancer antigen 125. Cancer 120(21), 3311-3319 (2014).

20 Hassan R, Kindler HL, Jahan T et al. Phase II clinical trial of amatuximab, a chimeric antimesothelin antibody with pemetrexed and cisplatin in advanced unresectable pleural mesothelioma. Clin. Cancer. Res. 20(23), 5927-5936 (2014).

21 Geldres C, Savoldo B, Hoyos V et al. T lymphocytes redirected against the chondroitin sulfate proteoglycan- 4 control the growth of multiple solid tumors both in vitro and in vivo. Clin. Cancer. Res. 20(4), 962-971 (2014).

22 Schuberth PC, Hagedorn C, Jensen SM et al. Treatment of malignant pleural mesothelioma by fibroblast activation protein-specific re-directed T cells. J. Transl. Med. 11, 187 (2013).

23 Morello A, Sadelain M, Adusumilli PS. Mesothelin-targeted CARs: driving T cells to solid tumors. Cancer Discov. 6(2), 133-146 (2015).

24 Moon EK, Carpenito C, Sun J et al. Expression of a functional CCR2 receptor enhances tumor localization and tumor eradication by retargeted human $\mathrm{T}$ cells expressing a mesothelin-specific chimeric antibody receptor. Clin. Cancer Res. 17(14), 4719-4730 (2011).

25 Wang E, Wang LC, Tsai CY et al. Generation of potent T-cell immunotherapy for cancer using DAP12-based, multichain, chimeric immunoreceptors. Cancer Immunol. Res. 3(7), 815-826 (2015)

26 Beatty GL, Haas AR, Maus MV et al. Mesothelin-specific chimeric antigen receptor mRNA-engineered $\mathrm{T}$ cells induce anti-tumor activity in solid malignancies. Cancer Immunol. Res. 2(2), 112-120 (2014). 